05

\title{
Особенности полиморфного превращения при нагреве и охлаждении кобальта
}

\author{
(C) Л.В. Спивак, ${ }^{1}$ H.Е. Щепина ${ }^{2}$ \\ ${ }^{1}$ Пермский государственный национальный исследовательский университет, \\ 614990 Пермь, Россия \\ ${ }^{2}$ Естественнонаучный институт Пермского государственного национального исследовательского университета, \\ 614990 Пермь, Россия \\ e-mail: Ispivak2@mail.ru, neshchepina@mail.ru
}

Поступило в Редакцию 20 ноября 2021 г.

В окончательной редакции 3 января 2022 г.

Принято к публикации 24 января 2022 г.

С помощью дифференциальной сканирующей калориметрии высокого разрешения проведено исследование закономерностей полиморфного $\alpha \leftrightarrow \beta$-превращения в поликристаллическом кобальте. Определены значения энергии активации $\alpha \rightarrow \beta$-превращения при нагреве кобальта, эффективных значений энтальпии и энтропии в зависимости от термической предыстории металла. На основании данных дифференциальной сканирующей калориметрии высказано предположение, что при скоростях нагрева меньше $20^{\circ} \mathrm{C} / \mathrm{min} \alpha \rightarrow \beta$-переход можно отнести к реализуемым по одному механизму фазовым превращениям первого рода, тогда как при охлаждении $\beta \rightarrow \alpha$-превращение осуществляется за счет протекания нескольких бездиффузионных механизмов фазовой трансформации, реализуемых в близких температурных интервалах. Осуществлено сопоставление полученных результатов с данными, полученными при дифференциальной сканирующей кало-

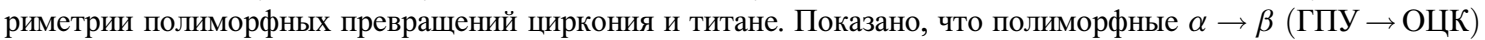
превращения в $\mathrm{Ti}$ и $\mathrm{Zr}$ имеют определенные отличия от перехода ГПУ $\rightarrow$ ГЦК в Со и не сводятся только к различному типу пространственной ориентации атомов (ОЦК или ГЦК).

Ключевые слова: энергия активации, калориметрия, полиморфизм, энтальпия, энтропия.

DOI: 10.21883/JTF.2022.04.52244.295-21

\section{Введение}

Считается, что к настоящему времени механизмы полиморфных превращений в чистых металлах хорошо изучены экспериментально и теоретически (см. [1-3]) и сформулированы некоторые общие, присущие данному явлению, закономерности. Как это часто бывает, появление более совершенных методов исследования позволяет вновь вернуться к анализу, казалось бы, уже хорошо известной аксиоматики. Это в полной мере относится к применению разработанной в последнее время технологии дифференциальной сканирующей калориметрии высокого разрешения (DSC). Однако при анализе научной литературы за последние 15 лет нам не удалось обнаружить проведенных другими авторами методом DSC исследований по изучению полиморфизма в таких хорошо известных металлах, как железо, титан, цирконий, кобальт, бериллий. Некоторым исключением следует считать детальное исследование методом DSC полиморфных превращений в уране [4]. Оказалось, что переходы $\alpha \rightarrow \beta \rightarrow \gamma($ ромбическая $\rightarrow$ тетрагональная $\rightarrow$ ОЦК) при нагреве урана контролируются диффузионными механизмами, а при охлаждении - близкими к бездиффузионным. Такая трактовка механизмов прямых и обратных полиморфных превращений в уране не совсем соответствует принятым в настоящее время представлениям. Относительно недавно были опубликованы работы по изучению методами DSC полиморфных превращений в железе, цирконии и титане [5-7]. В них показано, что в железе при полиморфном превращении соблюдаются общие закономерности $\alpha \leftrightarrow \gamma$-фазовой трансформации. Однако в цирконии, как и в титане, механизмы фазовых переходов ГПУ $\leftrightarrow$ ОЦК при нагреве отличаются от таковых при охлаждении. Вопрос о том, насколько эти особенности являются характерными и для полиморфных превращений типа ГПУ времени исследован не был. Это и определило изучение в настоящей работе методами DSC прямого и обратного $\alpha \leftrightarrow \beta$-превращений в поликристаллическом кобальте. Тем более, что относительно полиморфного ГПУ $\leftrightarrow$ ГЦК превращения в Со имеются неоднозначные данные о температурах $\alpha \leftrightarrow \beta$-превращений (см., например, [8]), величине температурного гистерезиса и энтальпии такого фазового перехода.

\section{1. Методика исследования}

Объектом исследования служили образцы, вырезанные из пластины толщиной $4 \mathrm{~mm}$ технически чистого электролитического кобальта $(99.92 \%$ Со). Данные по дифференциальной сканирующей калориметрии получены с помощью прибора STA „Јupiter“ 449 фирмы Netzsch. Нагрев и охлаждение производили в атмосфере аргона (99.9995\% Ar). Скорость потока газа 
25-30 ml/min. Масса образцов колебалась в пределах 180-210 mg. Обработка экспериментальных данных по DSC реализована с использованием программного обес-

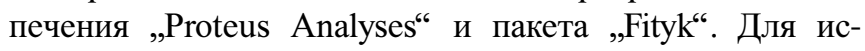
ключения возможности появления при исследовании фазовых превращений артефактов, обусловленных особенностями эксперимента, проводили сглаживание экспериментальных данных полиномом 6-8 степени.

Каждый термоцикл начинался с одного и того же исходного состояния кобальта.

В первом варианте это был нагрев одного отдельного образца со скоростью $40^{\circ} \mathrm{C} / \mathrm{min}$ до $250^{\circ} \mathrm{C}$ и выдержке при этой температуре $5 \mathrm{~min}$. После чего осуществлялись на том же образце последовательно термоциклы $\left(250 \leftrightarrow 550^{\circ} \mathrm{C}\right)$ со скоростями нагрева и охлаждения 5, 10,20 и $40^{\circ} \mathrm{C} / \mathrm{min}$.

Во втором варианте для каждой скорости нагрева использовался индивидуальный образец. Производили его нагрев со скоростью $40^{\circ} \mathrm{C} / \mathrm{min}$ до $250^{\circ} \mathrm{C}$ и выдерживали при этой температуре $5 \mathrm{~min}$, после чего осуществлялся его нагрев и охлаждение со скоростью или 5, или 10 , или 20 , или $40^{\circ} \mathrm{C} / \mathrm{min}$ для каждого отдельного образца. При этом изучалось влияние числа таких термоциклов $\left(250 \leftrightarrow 550^{\circ} \mathrm{C}\right)$ на термодинамические характеристики полиморфных превращений.

\section{2. Экспериментальные результаты и их обсуждение}

В первой серии экспериментов один и тот же образец последовательно нагревали и охлаждали со скоростями 5-10-20-40 $\mathrm{C} / \mathrm{min}$. Результаты одного их таких экспериментов представлены на рис. 1 .

Изменение сигнала DSC при нагреве и охлаждении и связанный с ним характер изменения второй производной сигнала DSC по температуре (DDDSC) имеют некоторый общий характер вне зависимости от схемы термоциклирования. Если при нагреве максимум DDDSC расположен внутри температурного интервала

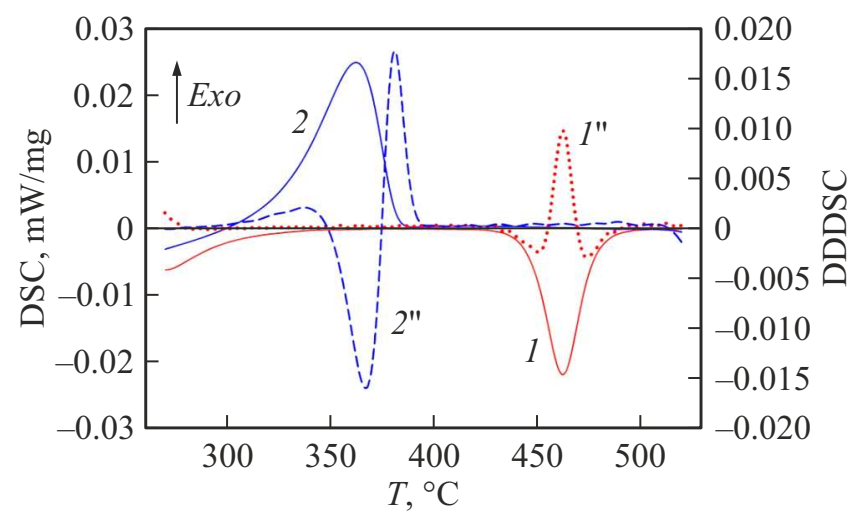

Рис. 1. Изменение сигнала DSC и вид второй производной (DDDSC) при нагреве $\left(1,1^{\prime \prime}\right)$ и охлаждении $\left(2,2^{\prime \prime}\right)$ Со со скоростью $5^{\circ} \mathrm{C} / \mathrm{min}$. развития эндотермического процесса, то при охлаждении в интервале проявления экзотермической реакции минимум на DDDSC отсутствует. Фиксируется заметный гистерезис $\left(70-80^{\circ} \mathrm{C}\right)$ между температурой начала превращения при нагреве и температурой начала превращения при охлаждении. Кроме того, если при нагреве можно достаточно уверенно определить температуру завершения $\alpha \rightarrow \beta$-перехода, то при охлаждении это не так однозначно.

Различие в протекании $\alpha \leftrightarrow \beta$-перехода в Со при нагреве и охлаждении хорошо видно, если представить эндотермические или экзотермические максимумы как суперпозицию наложения нескольких подпиков (рис. 2).

Если при нагреве с небольшими скоростями экспериментальные данные достаточно удовлетворительно аппроксимируется одним пиком - Gaussian 1D, то при охлаждении ситуация совсем иная. Экзотермический максимум удается аппроксимировать только несколькими подпиками (рис. 2, $b$ ). Это свидетельствует, повидимому, о том, что регистрируется протекание двух различных механизмов зарождения центров новой фазы с близкими температурами своей реализации. Во всех таких случаях имеется подпик $\left(P_{3}\right)$, отражающий процессы, происходящие во всем температурном интервале фазового превращения.

При нагреве со скоростями, превышающими $10^{\circ} \mathrm{C} / \mathrm{min}$, при аппроксимации эндотермического эффекта наблюдается еще один подпик $\left(P_{1}\right)$, прилегающий к левому фронту эндотермического максимума (рис. 3,a). Структура экзотермического пика при охлаждении сохраняется (рис. $3, b)$.

Данные DSC-зависимости при регистрации эндотермических или экзотермических эффектов по формальному признаку могут быть отнесены к фазовому переходу I рода, контролируемого одним механизмом фазовой трансформации, если различие температур максимума (минимума) второй производной сигнала DSC по температуре DDDSC и минимума (максимума) калориметрического эффекта отличаются не более, чем на $0.1-0.3^{\circ} \mathrm{C}([9,10])$. Этому условию в нашем случае отвечает только нагрев со скоростью $5^{\circ} \mathrm{C} / \mathrm{min}$. C увеличением скорости нагрева это различие увеличивается и для скорости нагрева $40^{\circ} \mathrm{C} / \mathrm{min}$ достигает $2^{\circ} \mathrm{C}$. Рис. $3, a$ иллюстрирует данную ситуацию. В полной мере это относится и к результатам аппроксимации экзотермических максимумов при охлаждении (рис. $2, b$ и $3, b$ ).

На рис. 4, $a$ показано влияние скорости нагрева на вид и расположение эндотермического пика при нагреве кобальта при первой схеме эксперимента.

По оси ординат отложена так называемая приведенная удельная теплоемкость $\left(C_{p_{\text {excess }}}\right.$ в соответствии с уравнением (1) (см. [11]), где $\dot{Q}_{S}$ и $\dot{Q}_{B l}-$ тепловые потоки при нагреве с образцом и базовыми тепловыми потоками соответственно, $m_{s}$ - масса образца, $\beta-$ скорость 

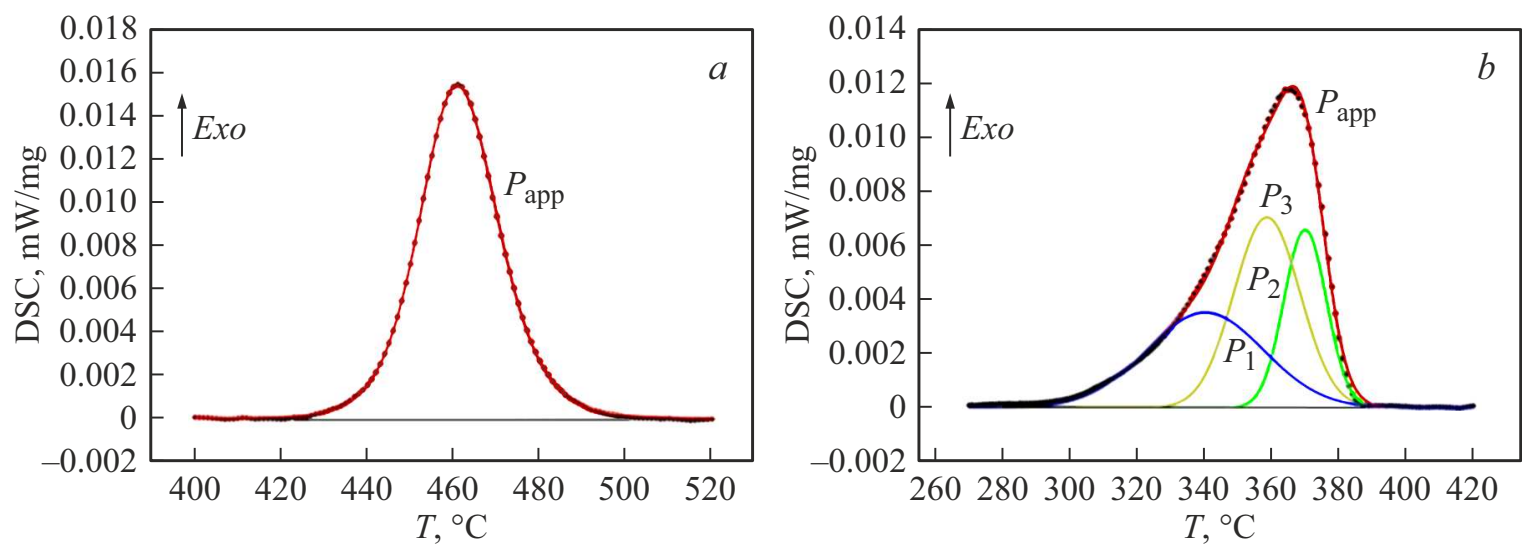

Pис. 2. Структура эндотермического пика при нагреве $(a)$ и экзотермического пика при охлаждении $(b)$ кобальта. Точки экспериментальные данные; $P_{a p p}-$ результат аппроксимации; $P_{1}, P_{2}$ и $P_{3}-$ подпики. Скорость термоциклирования $-5^{\circ} \mathrm{C} /$ min.
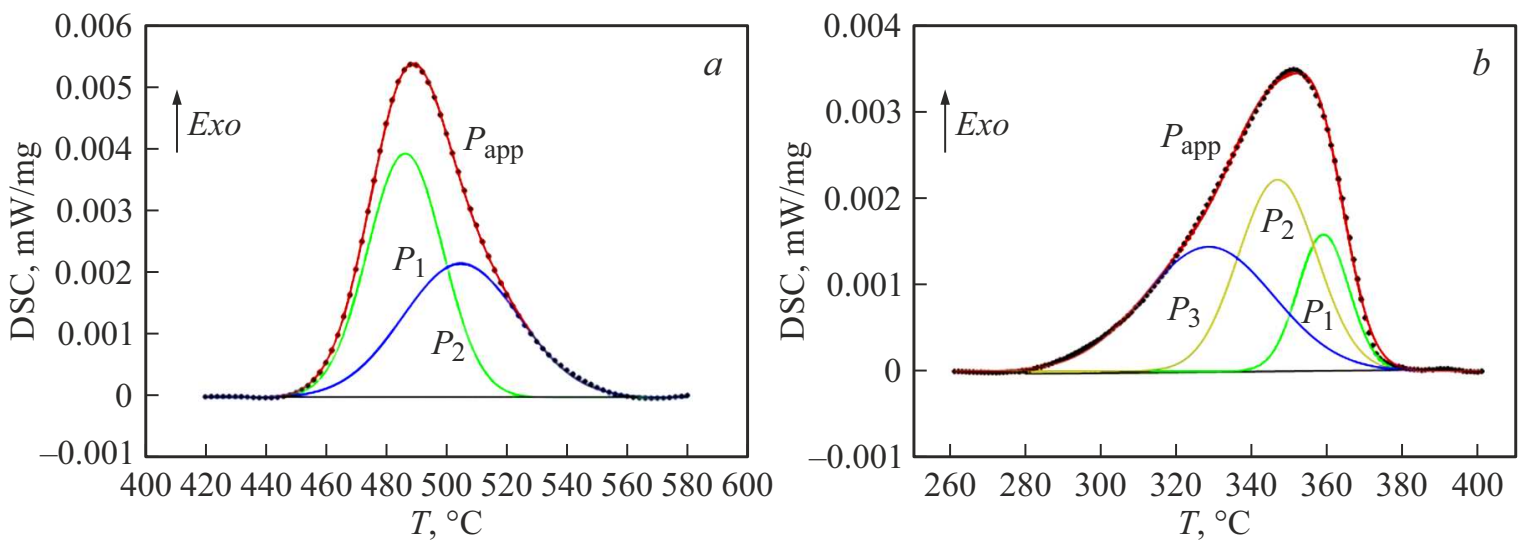

Pис. 3. Структура эндотермического пика при нагреве $(a)$ и экзотермического пика при охлаждении $(b)$ кобальта. Точки экспериментальные данные; $P_{a p p}-$ результат аппроксимации; $P_{1}, P_{2}$ и $P_{3}-$ подпики. Скорость термоциклирования $-40^{\circ} \mathrm{C} / \mathrm{min}$ Последовательный нагрев.
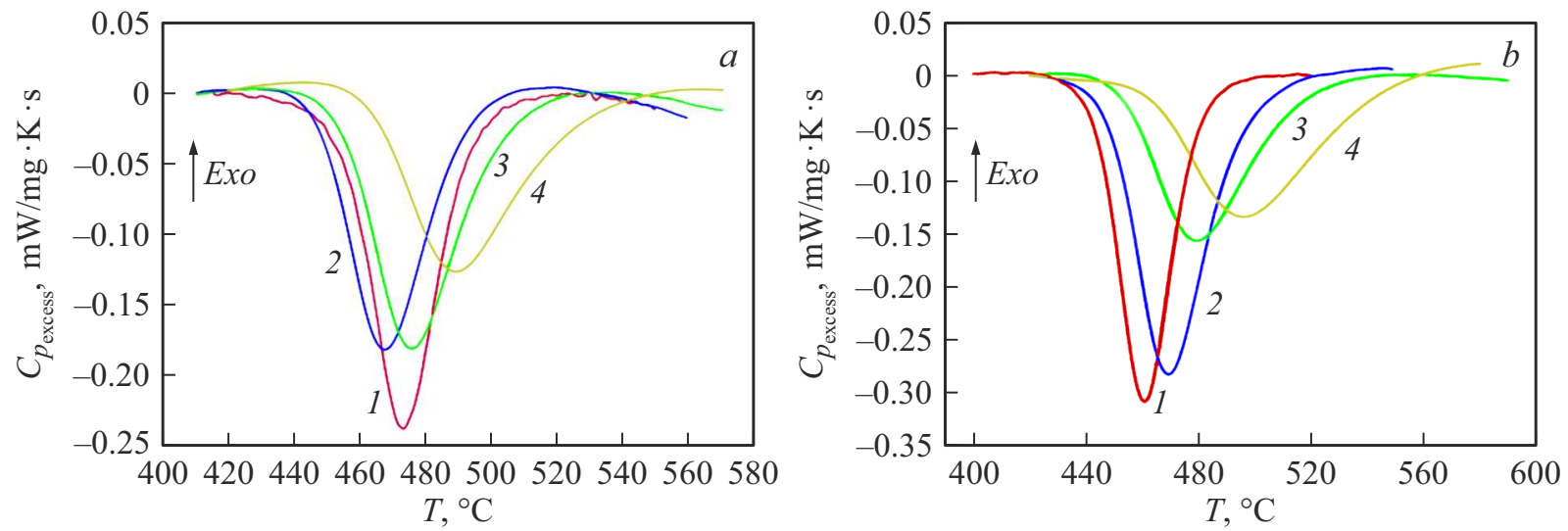

Рис. 4. Влияние скорости нагрева на вид и расположение эндотермических пиков при $\alpha \rightarrow \beta$-превращении в Со. $1-5,2-10$, $3-20,4-40^{\circ} \mathrm{C} / \mathrm{min} ; a-$ последовательный нагрев одного и того же образца; $b-$ индивидуальный нагрев образцов (второй цикл).

нагрева, $\left[{ }^{\circ} \mathrm{C} / \mathrm{s}\right]$.

$$
C_{p_{\text {excess }}}=\frac{\dot{Q}_{s}-\dot{Q}_{B l}}{m_{s} \beta}\left(\mathrm{Jg}^{-1} \cdot \mathrm{K}^{-1}\right)
$$

При этом наблюдается некоторая особенность, заключающаяся в том, что температура минимума эндотермического пика при скорости нагрева $5^{\circ} \mathrm{C} / \mathrm{min}$ оказывает- 


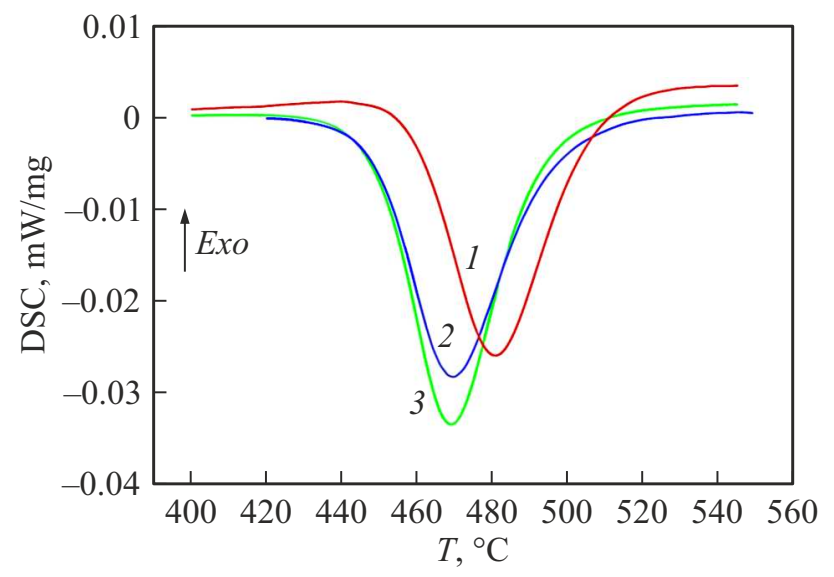

Рис. 5. Влияние числа циклов нагрева на вид и расположение эндотермического максимума при $\alpha \rightarrow \beta$-превращении в Со. Скорость нагрева $-10^{\circ} \mathrm{C} / \mathrm{min} .1-$ первый нагрев; $2-$ второй нагрев; 3 - третий нагрев.

ся несколько выше соответствующей температуры при последующем нагреве с большей скоростью (рис. 4,a). Так, например, для скорости нагрева $5^{\circ} \mathrm{C} / \mathrm{min}$ она равна $472.6^{\circ} \mathrm{C} ; 10^{\circ} \mathrm{C} / \mathrm{min}-467.2^{\circ} \mathrm{C} ; 20^{\circ} \mathrm{C} / \mathrm{min}-475.2^{\circ} \mathrm{C}$; $40^{\circ} \mathrm{C} / \mathrm{min}-488.8^{\circ} \mathrm{C}$. Тепловой эффект превращения в этом варианте термоциклирования слабо зависит от скорости нагрева и равен $3.4 \pm 0.1 \mathrm{~J} / \mathrm{g}$. То есть эффективное значение энтальпии оценивается в данном случае как $\Delta H^{\prime}=0.20 \pm 0.01 \mathrm{~kJ} / \mathrm{mol}$. Это много меньше величины энтальпии, полученной методом импульсного нагрева [12]: $0.45 \pm 0.03 \mathrm{~kJ} / \mathrm{mol}$. Значение конфигурационной энтропии $\Delta S^{\prime}$ лежит в интервале значений $0.25-0.28 \mathrm{~kJ} /(\mathrm{mol} \cdot \mathrm{K})$ или $\Delta S^{\prime}=0.27 \pm 0.01 \mathrm{~J} /(\mathrm{mol} \cdot \mathrm{K})$.

При реализации второй схемы эксперимента для каждой скорости нагрева использовался новый образец в исходном состоянии, т.е. состоянии при поставке. На каждом таком образце производилось последовательно три цикла „нагрев-охлаждение с постоянной скоростью“. Данные для одного из таких экспериментов представлены на рис. 5. Как видно из рис. 5, второй и третий циклы дают достаточно близкие результаты по изменению сигнала DSC при нагреве, тогда как при первом нагреве минимум на DSC расположен при более высоких температурах. Это справедливо для всех скоростей нагрева.

Что касается теплового эффекта превращения, то для одной и той же ситуации типичны следующие средние по трем нагревам значения. Для скорости нагрева $5^{\circ} \mathrm{C} / \mathrm{min}: 5.3 \pm 0.5 \mathrm{~J} / \mathrm{g} ; 10^{\circ} \mathrm{C} / \mathrm{min}: 5.6 \pm 0.3 \mathrm{~J} / \mathrm{g}$; $20^{\circ} \mathrm{C} / \mathrm{min}: 4.0 \pm 0.3 \mathrm{~J} / \mathrm{g} ; 40^{\circ} \mathrm{C} / \mathrm{min}: 4.1 \pm 0.3 \mathrm{~J} / \mathrm{g}$. Первому нагреву отвечает обычно наибольшее значение теплового эффекта.

Тот факт, что с увеличением числа циклов энергетические затраты на $\alpha \rightarrow \beta$-переход уменьшаются, позволяет считать, что в данном случае реализуется некий механизм наследственности, при котором в какой-то мере воспроизводится траектория смещения атомов при переходе от ГПУ структуры к ГЦК структуре. В этом наблюдается некоторый элемент памяти, отмеченный ранее для последовательной схемы термоциклирования.

При второй схеме эксперимента влияние скорости нагрева на структуру эндотермических и экзотермических минимумов и максимумов в качественном плане аналогично тому, как это установлено для последовательного нагрева одного и того же образца.

Влияние скорости нагрева на изменение сигнала DSC при $\alpha \rightarrow \beta$-превращении в Со для второй схемы эксперимента представлено на рис. $4, b$. Смещение температуры максимума скорости эндотермического процесса в область более высоких температур с увеличением скорости нагрева позволяет оценить методом Киссинджера [13] энергию активации $(Q)$ полиморфного превращения в Со при переходе $\alpha \rightarrow \beta$ :

$$
Q=-R \frac{\ln \left(\frac{\beta_{1}}{T_{1}^{2}}\right)-\ln \left(\frac{\beta_{2}}{T_{2}^{2}}\right)}{\left(\frac{1}{T_{1}}-\frac{1}{T_{2}}\right)},
$$

где $Q-$ энергия активации, $[\mathrm{kJ} / \mathrm{mol}] ; R-$ газовая постоянная; $T_{1}$ и $T_{2}$ - температуры максимумов на зависимости сигнала DSC при нагреве; $\beta_{1}$ и $\beta_{2}-$ скорости нагрева.

При построении графика зависимости величины $\ln \left(\beta / T_{\max }^{2}\right)$ от обратной температуры максимума на кривой сигнала $\operatorname{DSC}\left(1 / T_{\max }\right)$ в идеальном случае экспериментальные точки должны лежать на одной прямой, тангенс угла наклона которой позволяет оценить величину энергии активации перехода $\alpha \rightarrow \beta$. На рис. 6, $a$ представлены в указанных координатах экспериментальные результаты, полученные для второго цикла нагрева, которые, как видно, нельзя аппроксимировать одной линейной зависимостью. В противном случае метод решения шести попарных уравнений Киссинджера приводит к большим погрешностям в определении средней величины $Q$, что и было продемонстрировано ранее (см. $[6,7])$.

Для сравнения на рис. $6, b$ представлены аналогичные данные для Ті [7]. Подобная ситуация характерна и для циркония (см. [6]).

Таким образом, информация, представленная на рис. $6, a$, позволяет утверждать, что энергия активации полиморфного превращения в Со ( $\mathrm{Ti}$ и $\mathrm{Zr}$ ) зависит от скорости нагрева. В такой постановке эксперимента для малых скоростей нагрева отвечает значение энергии активации полиморфного превращения 370-380 kJ/mol. Для более высоких скоростей - 230-240 kJ/mol. Это справедливо для первого, второго и третьего циклов нагрева. Нечто подобное наблюдалось в [14], правда, объектом исследования там были сплавы с эффектом памяти формы.

Сама зависимость энергии активации фазового перехода от скорости нагрева, скорее всего, обусловлена сменой механизмов перехода от одной аллотропической 

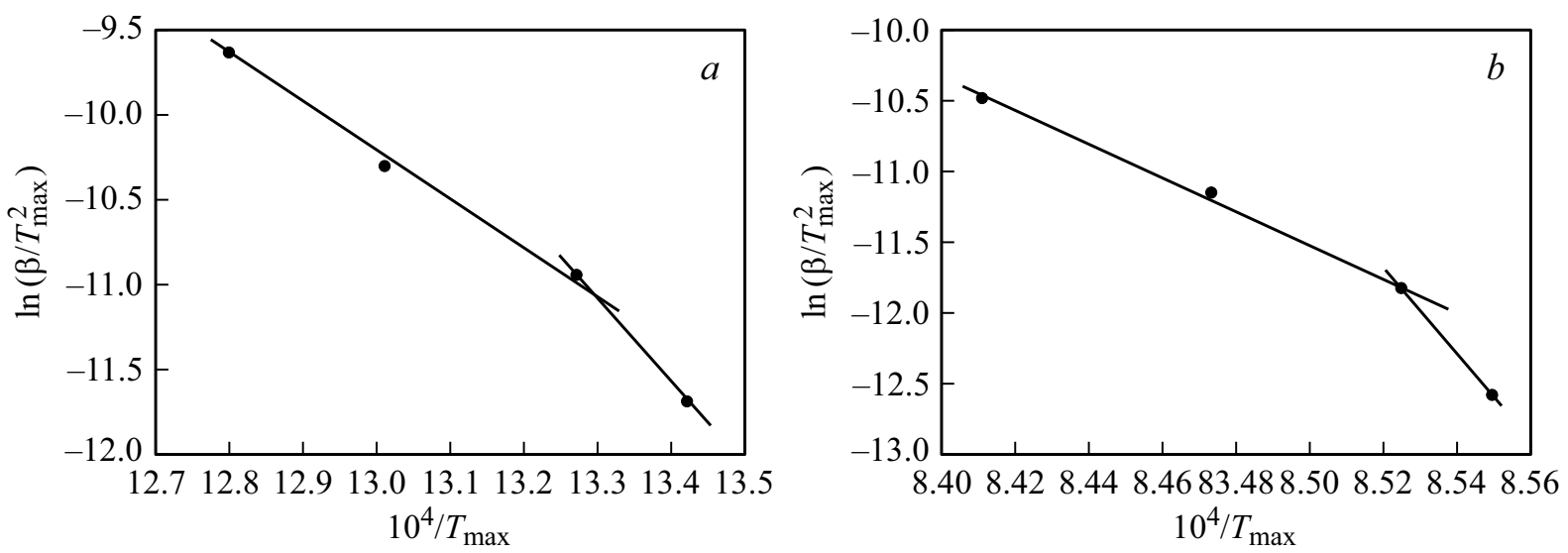

Рис. 6. Зависимость величины $\ln \left(\beta / T_{m}^{2}\right)$ от обратной температуры расположения эндотермического максимума при нагреве образцов Со $(a)$ и Ті $(b)$. Индивидуальный нагрев. Второй цикл.

формы Со к другой. И это опосредовано коррелирует с результатами аппроксимации эндотермических максимумов практически одним пиком (малая скорость нагрева) или двумя подпиками (большие скорости нагрева).

Эти данные так же подтверждают тенденцию, согласно которой с увеличением скорости нагрева энергетические затраты на $\alpha \rightarrow \beta$-превращение уменьшаются.

По аналогии с полиморфным превращением $\alpha \rightarrow \gamma$ в железе (см. [5,6]), при низких скоростях нагрева в Со $\alpha \rightarrow \beta$-превращение реализуется преимущественно (см. также [14]) по бездиффузионному механизму (массивное превращение). С увеличением скорости нагрева термодинамический стимул превращения и диффузионная подвижность атомов Со возрастают. Это обеспечивает возникновение и достаточно быстрый рост вновь возникающих кристаллитов. С увеличением скорости нагрева дифференциация подпиков при аппроксимации эндотермических эффектов становится более заметной.

При охлаждении, когда велика разница в температурах начала превращения при нагреве и начала превращения при охлаждении $\left(70-80^{\circ} \mathrm{C}\right)$ и, учитывая относительно низкую диффузионную активность атомов Со при невысокой температуре, $\beta \rightarrow \alpha$-превращения $\left(400-200^{\circ} \mathrm{C}\right)$ происходят по различным вариантам бездиффузионного фазового перехода. Действительно, электронно-микроскопические исследования указывают на возможность существования в Со трех типов мартенситных превращений (см. [15-19]): $\varepsilon$ (ГПУ), $\varepsilon^{\prime}$ (4Н или двойная ГПУ структура) и $\varepsilon^{\prime \prime}$ (9R или орторомбическая структура). Видимо, с этим связана аппроксимация экзотермического процесса в этой области температур (рис. $2, b$ и $3, b$ ) как суперпозиции трех подпиков. Как при нагреве, так и при охлаждении подпик, расположенный во всем температурном интервале наблюдения экзо- или эндотермических эффектов, по-видимому, обусловлен ростом возникающих кристаллов новой фазы. Другие подпики отражают процесс возникновения новых кристаллов.
Интересно сравнить полученные при исследовании с применением DSC данные по кобальту с данными, полученными с применением той же методики по цирконию и титану (см. $[6,7])$. Это сопоставление проведено для индивидуального нагрева со скоростью $5^{\circ} \mathrm{C} / \mathrm{min}$, как скорости, обеспечивающей наиболее близкое приближение к термодинамически равновесному состоянию.

Для Со тепловой эффект $\alpha \rightarrow \beta$-превращения равен $5.4 \pm 0.2 \mathrm{~J} / \mathrm{g}$; эффективное значение энтальпии $\Delta H^{\prime}=0.32 \mathrm{~kJ} / \mathrm{mol}$, эффективное значение конфигурационной энтропии $\Delta S^{\prime}=0.43 \mathrm{~J} /(\mathrm{mol} \cdot \mathrm{K})$.

Для Тi соответственно $40 \pm 4 \mathrm{~J} / \mathrm{g} ; \Delta H^{\prime}=1.9 \mathrm{~kJ} / \mathrm{mol}$, $\Delta S^{\prime}=1.6 \mathrm{~J} /(\mathrm{mol} \cdot \mathrm{K})$.

Для $\mathrm{Zr}$ соответственно $23 \pm 2.6 \mathrm{~J} / \mathrm{g}, \Delta H^{\prime}=2.1 \mathrm{~kJ} / \mathrm{mol}$, $\Delta S^{\prime}=1.8 \mathrm{~J} /(\mathrm{mol} \cdot \mathrm{K})$.

Как видно из приведенных данных, различие между металлами с переходом ГПУ $\rightarrow$ ОЦК и ГПУ $\rightarrow$ ЦЦК довольно существенное. Отсюда следует, что такой переход может осуществляться различными механизмами.

Закономерности $\alpha \rightarrow \beta$ (ГПУ $\rightarrow$ ОЦК) превращения имеют определенные отличия от перехода ГПУ $\rightarrow$ ГЦК. В первую очередь, к ним следует отнести следующее.

При всех скоростях нагрева в Со максимум DDDSC лежит в интервале температур регистрации эндотермического эффекта. В цирконии и титане это наблюдается лишь при нагреве со скоростями выше $10^{\circ} \mathrm{C} / \mathrm{min}$.

При охлаждении Со в районе температур реализации $\beta \rightarrow \alpha$-превращения максимума на DDDSC не наблюдается. Экзотермический эффект можно представить как суперпозицию трех подпиков.

В титане и цирконии с увеличением скорости термоциклирования такой экстремум на кривых DDDSC появляется как при нагреве, так и при охлаждении. Экзотермический эффект при охлаждении хорошо аппроксимируется двумя подпиками (SplitGaussian).

Термоциклирование при постоянной скорости в титане и цирконии подавляет тепловой эффект при $\alpha \leftrightarrow \beta$ превращении, тогда как в кобальте подобное явле- 
ние не зарегистрировано при всех скоростях термоциклирования.

Температурный гистерезис между началом превращения при нагреве и началом превращения при охлаждении в кобальте в несколько раз больше, чем в титане и цирконии. Последнее является косвенным признаком реализации мартенситного механизма превращения.

\section{Выводы}

Полиморфное превращение $\alpha \leftrightarrow \beta$ в Со реализуется при нагреве и охлаждении по различным механизмам атомной перегруппировки. И если при нагреве такая трансформация близка к фазовому переходу первого рода, осуществляемому по одному механизму фазового превращения, то при охлаждении она представляет собой наложение калориметрических эффектов от нескольких превращений, реализуемых в близких температурных интервалах.

Полиморфные $\alpha \rightarrow \beta$ (ГПУ $\rightarrow$ ОЦК) превращения в Ti и $\mathrm{Zr}$ имеют определенные отличия от перехода ГПУ $\rightarrow$ ГЦК в Со и не сводятся только к различному типу пространственной ориентации атомов (ОЦК или ГЦК). Как показано выше, они связаны с термодинамическими и кинетическими особенностями такой фазовой трансформации.

Полиморфное превращение при нагреве $\mathrm{Ti}, \mathrm{Zr}$ и $\mathrm{Co}$ характеризуется зависимостью энергии активации такого перехода от скорости нагрева.

Для исследованных металлов с ГПУ решетками их поведение при нагреве и охлаждении в районе температур полиморфного превращения в той или иной мере всегда зависит от термической предыстории конкретного объекта исследования.

\section{Конфликт интересов}

Авторы заявляют, что у них нет конфликта интересов.

\section{Список литературы}

[1] Я.С. Уманский, Ю.А. Скаков. Физика металлов (Атомиздат, М., 1978)

[2] R. Abbaschian, L. Abbaschian, R.E Reed-Hill. Physical Metallurgy. Principles (Cengage Learning ( ), 2009)

[3] G.N. Haidemenopoulos. Physical Metallurgy. Principles and Design (CRC Press-Taylor and Francis 2018), DOI: $10.1201 / 9781315211220$

[4] A.K. Rai, S. Raju, B. Jeyaganesh, E. Mohandas, R. Sudha, V. Ganesan. J. Nucl. Mater., 383, 215 (2009).

[5] С.А. Оглезнева, Л.В. Спивак, М.Н. Каченок, М.Н. Порталов. Металлы, 2, 91 (2015).

[6] Л.В. Спивак, Н.Е. Щепина. ЖТФ, 90 (7), 1145 (2020). DOI: $10.21883 /$ JTF.2020.07.49449.381-19

[7] Л.В. Спивак, Н.Е. Щепина. ЖТФ, 91 (8), 1233 (2021). DOI: $10.21883 /$ JTF.2021.08.51096.53-21s

[8] W. Betteridge. Progr. in Mater. Sci., 24, 51 (1980).
[9] P.J. Van Ekeren. Handbook of Thermal Analysis and Calorimetry Vol. 1: Principles and Practice, M.E. Brown, editor. (Elsevier Science B., 75, 1998)

[10] В.А. Алешкевич. Молекулярная физика (Физматлит, М., 2016)

[11] S.M. Sarge, G.W.H. Höhne, W.F. Hemminger. Calorimetry. Fundamentals Instrumentation and Applications (WileyVCH Verlag GmbH \& Co. KGaA: Weinheim, Germany, 2014)

[12] A.S. Dobrosavljevic, K.D. Maglic, N.L. Perovic. High Temp.High Pressures, 21 (3), 317 (1989).

[13] H.E. Kisinger. Analyt. Chem., 29, 1702 (1957).

[14] J.C. Zhao, M.R. Notis. Scripta Metall. Mater., 32 (10), 1671 (1995).

[15] Z.N. Zhou, L. Yang, R.C. Li, J.-G. Li. Intermetallics, 92, 49 (2018).

[16] F. Cardellinia, G. Mazzonea. Phil. Mag. A., 67 (6), 1289 (1993).

[17] A. Muniera, J.E. Bidauxa, R. Schallera, C. Esnoufa. J. Mater. Res., 5 (4), 769 (1990).

[18] A.E. Ray, S.R. Smith. J. Phase Equilibria, 12 (6), 644 (1991).

[19] H. Matsumoto. J. Alloys Compounds, 223 (1), 11 (1995). 